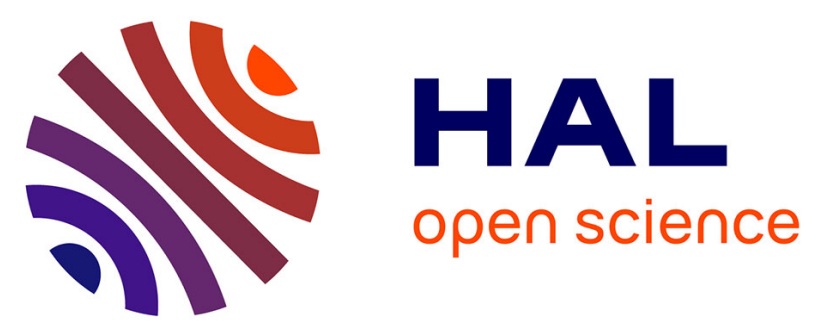

\title{
Analysis of time-resolved laser-induced breakdown spectra by mean field-independent components analysis (MFICA) and multivariate curve resolution-alternating least squares (MCR-ALS)
}

Maria El Rakwe, Douglas Neil Rutledge, Gilles Moutiers, Jean-Baptiste Sirven

\section{To cite this version:}

Maria El Rakwe, Douglas Neil Rutledge, Gilles Moutiers, Jean-Baptiste Sirven. Analysis of timeresolved laser-induced breakdown spectra by mean field-independent components analysis (MFICA) and multivariate curve resolution-alternating least squares (MCR-ALS). Journal of Chemometrics, 2017, 31 (2), pp.e2869. 10.1002/cem.2869 . cea-02388593

\section{HAL Id: cea-02388593 \\ https://hal-cea.archives-ouvertes.fr/cea-02388593}

Submitted on 23 Jan 2020

HAL is a multi-disciplinary open access archive for the deposit and dissemination of scientific research documents, whether they are published or not. The documents may come from teaching and research institutions in France or abroad, or from public or private research centers.
L'archive ouverte pluridisciplinaire $\mathbf{H A L}$, est destinée au dépôt et à la diffusion de documents scientifiques de niveau recherche, publiés ou non, émanant des établissements d'enseignement et de recherche français ou étrangers, des laboratoires publics ou privés. 


\title{
Analysis of time-resolved laser-induced breakdown spectra by mean field-independent components analysis (MFICA) and multivariate curve resolution-alternating least squares (MCR-ALS)
}

\author{
Maria El Rakwe $^{1}$ | Douglas Neil Rutledge ${ }^{2}$ | Gilles Moutiers ${ }^{1}$ | Jean-Baptiste Sirven ${ }^{1}$ \\ ${ }^{1}$ CEA, DEN, DPC, SEARS, LANIE, Gif-sur- \\ Yvette, France \\ ${ }^{2}$ AgroParisTech, UMR 1145 IngénierieProcédés \\ Aliments, Paris, France
}

Laser-induced breakdown spectroscopy (LIBS) is an analytical technique allowing the determination of elemental concentrations in a variety of matrices in the solid, liquid, and gaseous phases. Because of the inherent complexity of the signal and to the high dimensionality of experimental data, chemometrics has been more and more applied in LIBS to perform samples identification or quantitative measurements. But multivariate methods can also be used for the description and physical interpretation of the plasma, particularly to exploit the temporal dimension of the LIBS signal, which is usually neglected in spectrochemical measurements. In this work, time-resolved spectra of a pure aluminum sample were treated with 2 methods, mean field-independent components analysis and multivariate curve reso-lution-alternating least squares, applying non-negativity constraints for scores and components in both cases. Results obtained were compared with reference univariate measurements of the emission of the species of interest (ions, neutral atoms, and molecules). The interpretation of scores and components provided a physical description of phenomena that take place between species in the plasma, like ionic recombination and molecules formation. Overall, mean field-independent compo-nents analysis and multivariate curve resolution-alternating least squares yield equivalent solutions with our dataset. This new approach is very promising for the treatment of time-resolved data obtained by LIBS.

\begin{abstract}
KEYWORDS
chemometrics, ion recombination, laser-induced breakdown spectroscopy, LIBS, MCR-ALS, mean field-independent components analysis, MFICA, molecular formation, multivariate curve resolution-alternating least squares, temporal evolution
\end{abstract}

\section{1 | INTRODUCTION}

Laser-induced breakdown spectroscopy (LIBS) is an analytical technique that allows the determination of a sample's elemental composition. A focused laser pulse induces a plasma at the

* Mean field-independent components analysis and multivariate curve resolution-alternating least squares were used for the first time to describe the temporal evolution of a laser-induced aluminum plasma emission. When non-negativity constraints were applied both for scores and components, both methods provided an equivalent description of phenomena that take place between the species, like ionic recombination and formation of molecules. This new approach is very promising for the treatment of time-resolved data obtained by laser-induced breakdown spectroscopy. sample surface, and the emission spectrum of atoms, ions, or molecules is detected and analyzed in the UV-visible range. The characteristics of LIBS, which include remote and rapid analysis, no sample preparation, applicability to any type of sample, and potential for field portability, make this quasi nondestructive analytical technique a very attractive method. ${ }^{1,2}$ Laser-induced breakdown spectroscopy has developed in many fields, such as the nuclear industry, ${ }^{3}$ aerosols analysis, ${ }^{4}$ cultural heritage,${ }^{5}$ biology, ${ }^{6}$ polymers, ${ }^{7}$ and metallurgy, ${ }^{8}$ and the number of applications is still growing. The LIBS signal stems from highly nonlinear, coupled phenomena driving the sample laser ablation and the laser-plasma interaction. It is 
multi-elemental, depends on time, and is a mixture of the emission of all species present in the plasma, which can interact with each other. From an instrumental point of view, LIBS spectra are of large dimension, up to several tens of thousands of channels, and can be acquired in a very short time. This inherent complexity of the LIBS signal, added to the high dimensionality of the data, has soon stimulated the use of multivariate data analysis methods to efficiently extract useful information from the experimental spectra.

Hence, very recently, Zhang et $\mathrm{al}^{9}$ reviewed the use of chemometric methods in qualitative and quantitative analysis by LIBS and in spectral data preprocessing. The data preprocessing methods in LIBS mainly consist of baseline correction, ${ }^{10,11}$ noise filtering, ${ }^{12,13}$ overlapping peak resolution, ${ }^{14}$ and data compression. ${ }^{15}$ Chemometric techniques have proven efficient for samples identification and classification by LIBS. To give only a few examples, principal components analysis (PCA) and independent components analysis (ICA) were successfully used for rocks discrimination. ${ }^{16,17}$ Partial least squares-discriminant analysis was extensively developed for security purposes (identification of explosives and chemical or biological warfare agents), ${ }^{18,19}$ as well as neural networks. ${ }^{15}$ Soft independent modeling of class analogy was used for the analysis of uranium concentrate ${ }^{20}$ and carbonate grains. ${ }^{21}$ Now, the comparison of performances of different techniques is a growing topic in LIBS, as shown by recent papers on the subject. ${ }^{22,23}$ In quantitation by LIBS, chemometric methods are used to cope with nonlinearities between the emission signal and the concentration and with matrix effects. Hence, partial least-squares regression is currently used to determine the elemental composition of Martian rocks measured by the LIBS instrument onboard the Curiosity rover, and more advanced techniques are being developed. ${ }^{24}$ Artificial neural networks were used for on-site quantitative analysis of soils, ${ }^{25}$ for calibration of chrome and nickel in steel, ${ }^{26}$ and to determine the concentration of nickel and vanadium in vacuum residues of crude oils. ${ }^{27}$ More recent techniques were also tested by different authors, such as support vector machines regression for slag samples analysis ${ }^{9}$ or random forest regression for steel analysis. ${ }^{8}$ In fact, so far chemometric methods have been exclusively used in LIBS data analysis to perform samples identification or for quantitative measurements, and it is now clear that in both fields, chemometrics is becoming an essential tool.

However, LIBS spectra do not only contain chemical but also physical information on the plasma. Some of its features, such as the electron temperature and density, which can be determined by standard spectroscopic methods, ${ }^{1}$ are key parameters to understand its underlying physics. The use of chemometrics in this field is in its infancy, but interesting applications have already been published, with important consequences for particular quantitation approaches. ${ }^{28,29}$ Among the different physical characteristics of the LIBS signal, one crucial feature is its time dependence. Indeed, LIBS measurements consist of time-resolved and spectrally resolved spectra of atomic and ionic lines emitted by the plasma, on a microsecond time scale. Typically, the time between the laser pulse and the start of the recording of the plasma emission (gate delay) and the integration time (gate width) are fixed for spectroscopic measurements. Similarly, for kinetics studies, certain spectral lines are selected, which can be a difficult task when dealing with complex spectra. Therefore, either the temporal or spectral dimension of the LIBS signal is usually neglected, although exceptions can be found. As an example, Bohling et al showed that the accuracy of identification of different materials is significantly improved when the decay times of certain lines of interest are added to the spectral intensity data as input variables of a neural network. ${ }^{15,30}$

Several chemometric tools like PCA, ICA, or multivariate curve resolution-alternating least squares (MCR-ALS) were successfully used by different authors to process timeresolved spectral data obtained by different techniques. Thus, an approach based on PCA for analyzing time-resolved X-ray absorption spectra was proposed by Smolentsev et al, which allowed the determination of the local structure of intermediate forms of organometallic compounds during reactions as well as the time dependence of their concentrations. ${ }^{31} \mathrm{By}$ analyzing the evolution of the mid infrared images as well as the changes of oxidized products over time, using PCA, Zhou et al proved that the dynamics of photooxidation of polyolefin could be described. ${ }^{32}$ Independent components analysis is becoming a method of choice to analyze temporally resolved data, such as in fluorescence spectroscopy, ${ }^{33}$ in functional magnetic resonance imaging, ${ }^{34}$ or in multichannel time-resolved absorption spectroscopy. For example, Martin et al have used ICA to find linear decompositions of multichannel time-resolved absorption spectroscopy datasets into physically meaningful components. ${ }^{35}$ Multivariate curve resolution-alternating least squares was applied to many types of data ${ }^{36}$ but also to study time-resolved data in Raman spectroscopy, ${ }^{37}$ in X-ray absorption spectroscopy, ${ }^{38}$ and in infrared and UV-visible spectroscopy. ${ }^{39,40}$ Ruckebusch et al used chemometrics (evolving factor analysis, MCR-ALS, and hard- and soft-MCR-ALS) to study time-resolved spectra in UV-visible femtosecond transient absorption spectroscopy, to identify an intermediate state of benzophenone, and to determine its spectrokinetic properties. ${ }^{41}$

In this work, we propose to use ICA and MCR-ALS to explore simultaneously both the temporal and spectral dimensions of the LIBS signal. Our fundamental objective is to determine the potential of those multivariate methods for the description and physical interpretation of the temporal evolution of spectra, particularly in light of the kinetics of ionic recombination and molecular formation within the plasma. We underline that our approach is intended as unsupervised and, as such, does not seek to incorporate any particular knowledge on the chemical system in the models. As mentioned later, only a non-negativity constraint for scores 
presupposing anything on the observed phenomena, was introduced both in ICA and in MCR-ALS. In doing this, we are aware that we do not use the MCR-ALS method at its full potential, but on the other hand, this allows a more straightforward comparison of its performances to those of ICA.

Thus, the temporal evolution of the LIBS signal of a pure aluminum sample was measured between 0.2 and 15 microseconds after the laser pulse. The meaning of extracted scores and components obtained by both techniques was then compared to the standard univariate approach and discussed.

\section{2 | EXPERIMENTAL}

\section{1 | Experimental setup}

The LIBS setup is the MobiLIBS equipment (Ivea Solution, France) using an Nd:YAG laser at $266 \mathrm{~nm}$ running at $20-\mathrm{Hz}$ repetition rate and providing 5-nanosecond (full width at half maximum) pulses. The maximum laser pulse energy was $3 \mathrm{~mJ}$, and the spot diameter at the sample surface was equal to $50 \mu \mathrm{m}$, leading to an irradiance of approximately $30 \mathrm{GW} \mathrm{cm}^{-2}$.

The emission from the plasma was collected in the direction of the incident laser beam and transmitted through an optical fiber to the entrance slit of an Echelle spectrometer (Mechelle, Andor Technology, resolving power $\lambda / \Delta \lambda=4000$ ) equipped with an intensified charge-coupled device camera (iStar, Andor Technology).

In this work, a pure aluminum sample (198f, TechLab, 99.99\% $\mathrm{Al}$ ) was analyzed. To be able to study the temporal behavior of the LIBS spectra, the delay time between the laser pulse and the signal acquisition varied within the range of 0.2 to 15 microseconds. The temporal gate width was adapted to different delay times to improve the signal intensity, which decreases when the plasma cools. Table 1 shows the gate delay and width used.

A total of 25 spectra were acquired for each delay in the spectral window between 200 and $900 \mathrm{~nm}$ (Figure 1). Each spectrum resulted from the accumulation of 50 laser shots. In addition, 2 pre-pulses were used to be able to remove the oxide layer $\left(\mathrm{Al}_{2} \mathrm{O}_{3}\right)$ that was naturally formed at the sample surface. $^{42}$

\section{2 | Spectral data}

The laser-induced plasma is inhomogeneous and transient because of its expansion and its fairly rapid cooling. Thus, the electron density and the plasma temperature undergo major variations over time.

Figure 1 shows the spectra measured at different gate delays after the laser pulse. We can see that the emission lines spectrum from the early stage of the plasma is superimposed on a relatively featureless continuous background emission because of Bremsstrahlung radiation (collisions between free electrons and excited atoms and ions) and radiative recombination of electrons with ions in the plasma. ${ }^{43}$
TABLE 1 Gate delay and width after the laser pulse used for spectra acquisition

\begin{tabular}{|c|c|}
\hline Gate delay, $\mu \mathrm{s}$ & Gate width, $\mu \mathrm{s}$ \\
\hline 0.2 & 0.1 \\
\hline 0.3 & 0.1 \\
\hline 0.4 & 0.1 \\
\hline 0.5 & 0.1 \\
\hline 0.6 & 0.1 \\
\hline 0.7 & 0.1 \\
\hline 0.8 & 0.1 \\
\hline 0.9 & 0.1 \\
\hline 1 & 0.1 \\
\hline 1.1 & 0.3 \\
\hline 1.4 & 0.3 \\
\hline 1.7 & 0.3 \\
\hline 2 & 0.5 \\
\hline 2.5 & 0.5 \\
\hline 3 & 0.5 \\
\hline 3.5 & 1 \\
\hline 4.5 & 1 \\
\hline 5.5 & 1.5 \\
\hline 7 & 3 \\
\hline 10 & 5 \\
\hline 15 & 10 \\
\hline
\end{tabular}

Then, following the decrease of the continuous radiation, first, the aluminum ionic lines are observed followed by neutral aluminum lines, and finally, molecular bands due to recombination of the chemical species. Since the continuous background and the elemental emission decay with different temporal rates, it is possible to use time-resolved detection to discriminate informative signals from the strong continuous radiation and also to avoid spectral interference between species that emit at different times during the plasma decay.

Also, it can be noted that the lines at 308.2 and $309.3 \mathrm{~nm}$ are reversed because of a very pronounced self-absorption, ie, absorption of photons emitted from the plasma core by colder atoms located at its periphery. This phenomenon is all the more pronounced that the element concentration in the plasma is higher, that the energy of the lower level of the transition is lower, and that the Einstein coefficient of the transition is higher. Therefore, it is most probable for resonant lines of major elements. When the delay increases, the lines sharpen and reversal is less marked.

A very marked broadening and a spectral shift for lines at 256.8 and $257.6 \mathrm{~nm}$ are observed, related to the Stark effect. This effect is the shifting and splitting of spectral lines due to the presence of an electric field caused by the free electrons present in the plasma in high density at short delay. The Stark effect can lead to splitting of degenerate energy levels. It increases with the plasma density.

It can also be seen that the lines at $308.2 \mathrm{~nm}\left(E_{\mathrm{k}}=4.02 \mathrm{eV}\right.$, $\left.g A=2.35 \mathrm{e}+08 \mathrm{~s}^{-1}\right)$ and $309.3 \mathrm{~nm}\left(E_{\mathrm{k}}=4.02 \mathrm{eV}\right.$, $g A=4.37 \mathrm{e}+08 \mathrm{~s}^{-1}$ ) last longer than the lines at $256.8 \mathrm{~nm}$ $\left(E_{\mathrm{k}}=4.82 \mathrm{eV}, g A=7.68 \mathrm{e}+07 \mathrm{~s}^{-1}\right)$ and $257.5 \mathrm{~nm}$ 

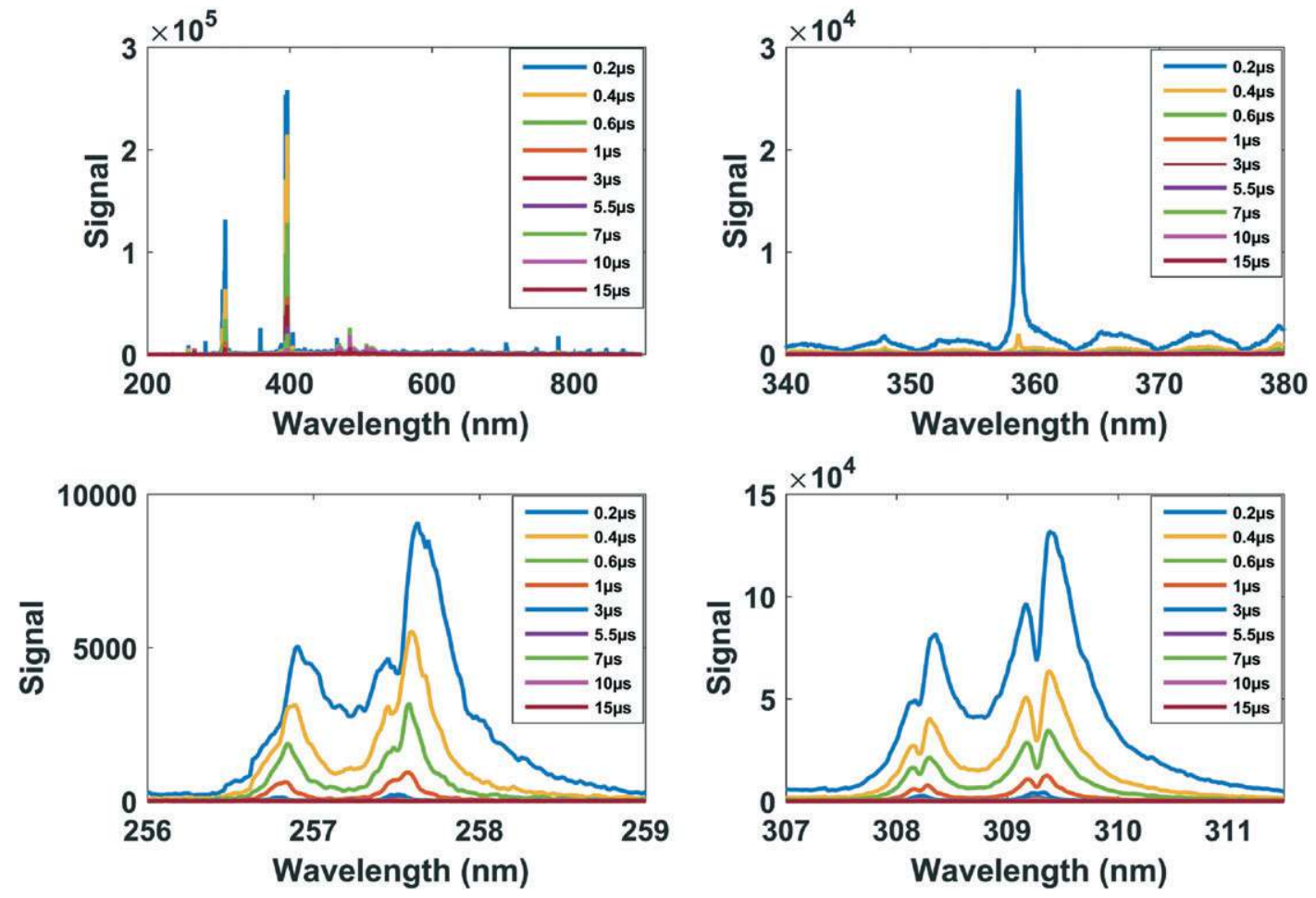

FIGURE 1 Spectra obtained at different gate delays after the laser shot

$\left(E_{\mathrm{k}}=4.82 \mathrm{eV}, 2.40 \mathrm{e}+07 \mathrm{~s}^{-1}\right)$ and this is because the upper energy level $\left(E_{\mathrm{k}}\right)$ and the $g A$ factor $(A$ : Einstein coefficient and $g$ : degeneracy level) of the latter lines are higher, and then they are harder to excite as the plasma cools down.

Therefore, the following lines, subject to a pronounced self-absorption leading to the line reversal, were eliminated from the dataset: $308.22,309.27,394.40$, and $396.15 \mathrm{~nm}$. The lines around 305 to $307 \mathrm{~nm}(305.01,305.47,305.71$, $305.90,305.99,306.43$, and $306.61 \mathrm{~nm}$ ) located on the edge of the 308.22-nm line were also eliminated. The $266-\mathrm{nm}$ laser line, visible on the spectra because of the persistence of the intensifier, was also removed. Except for this removal of some spectral regions, no preprocessing was applied to the spectra before chemometric treatment. The final dataset was composed of 525 spectra of 19564 wavelength channels.

\section{3 | CHEMOMETRIC METHODS}

Two chemometric tools were applied in this study: mean fieldindependent components analysis (MFICA) and MCR-ALS. These tools are designed to decompose a spectrum into a linear combination of synthetic spectra called components. The coefficients of the linear combination are called scores. The difference between the 2 methods lies in the criteria and constraints used in the calculation.

\section{1 | Independent components analysis}

Independent components analysis is a statistical and computational technique for extracting source signals from their

mixtures. The observed signals are considered as weighted sums of pure source signals, the weights being proportional to the contribution of the corresponding pure signals to each mixture. ${ }^{44,45}$ Therefore, the objective of ICA is to search for the least Gaussian possible sources, ie, the most independent. ${ }^{46-49}$ A number of ICA algorithms are to be found in the study of Krishnaveni et al. ${ }^{49}$ In our case, we used the MFICA algorithm. ${ }^{50}$ The particularities of MFICA are the non-negativity of components and weights, its appealing convergence, and high computational speed for high dimensional data. Compared to other algorithms, MFICA is a Bayesian iterative algorithm that can constrain both sources and the mixing matrix to be positive.

In MFICA, the source profiles are estimated from the mean of their posterior distribution, and the mixing matrix and noise level are estimated by maximizing a posterior solution. In MFICA, we applied a non-negativity constraint on both concentration and spectral profiles to be able to increase the model interpretability. This was applied using non-negative matrix factorization. ${ }^{51}$ In this case, the independence assumption is not totally satisfied, and so the resolved components are considered to be least dependent components. ${ }^{52}$

The optimal number of ICs was calculated by the ICAby-blocks algorithm. ${ }^{53}$

\section{2 | Multivariate curve resolution-alternating least squares}

Multivariate curve resolution-alternating least squares is an iterative multivariate self-modeling curve resolution method that aims to recover the response profile of pure components 
in an unresolved and unknown mixture obtained from evolutionary processes. ${ }^{54}$ The MCR-ALS methods try to maximize the data variance explained by the different components under a set of optional constraints (non-negativity, unimodality, closure, selectivity, local rank, and trilinearity).

Although different constraints could have been introduced, for example, an empirical kinetic model of ion recombination and molecules formation, we only imposed the non-negativity of the concentration and spectral profiles. Indeed, to be able to have as general an approach as possible, we aim at doing as unsupervised an analysis as possible. From that point of view, the non-negativity constraint does not introduce any particular knowledge on the system under study in the model, as it has a clear physical sense in all cases. In addition, in doing so, the comparison of MCR-ALS results to those of MFICA is more straightforward, since the same constraints are used for both methods.

The optimal number of components was determined by applying singular value decomposition. ${ }^{55,56}$ Once the number of components is estimated, the data structure can be analyzed using evolving factor analysis, ${ }^{57}$ which provides an initial estimation of components and proportions, which are optimized iteratively by an alternating least squares procedure. During the ALS optimization, convergence is achieved when in 2 consecutive iterative cycles, relative differences in standard deviations of the residuals between experimental and ALS calculated data values are less than a previously selected value. The MCR-ALS method is easily extended to simultaneous analysis of several data matrices. If the number and nature of columns (wavelengths in our case) is the same for all the data matrices, the analysis can be performed simultaneously over more than one data matrix.

Data treatment was done using MATLAB R2015a (The Math Works, Natick, USA). The MFICA method was obtained from the ICA:DTU Toolbox for MATLAB v3. ${ }^{58}$ The MCR-ALS toolbox was downloaded from the website. ${ }^{59}$

\section{4 | RESULTS}

In both cases, 3 components were found sufficient to describe the dataset. Information regarding extracted signals is gathered in Table 2, listing the Einstein coefficients $(A)$, the degeneracy levels $(g)$, and the lower and upper energy level values ( $E_{\mathrm{i}}$ and $E_{\mathrm{k}}$, respectively). The scores are individually normalized by the width of the measuring gate (see Table 1). They are compared with the reference univariate measurement, detailed later.

Figure 2 displays the components and scores obtained by MFICA. The first component (Figure 2A) shows ionic and neutral aluminum lines with the atomic lines of oxygen and nitrogen. It is the only component where the oxygen, nitrogen, and aluminum ionic lines appear significantly. In Figure 2B, MFICA only shows the neutral Al lines, so this component is associated with $\mathrm{Al}$ neutral atoms. The third
TABLE 2 Wavelength $(\lambda)$, degeneracy level $(g)$, Einstein coefficients $(A)$, lower and upper energy level values $\left(E_{\mathrm{i}}, E_{\mathrm{k}}\right)$ of the observed lines

\begin{tabular}{|lccc}
\hline Wavelengths of Al lines, $\mathbf{n m}$ & $\boldsymbol{E}_{\mathbf{i}}, \mathbf{e V}$ & $\boldsymbol{E}_{\mathbf{k}}, \mathbf{e V}$ & $\boldsymbol{g A}, \mathbf{s}^{\mathbf{- 1}}$ \\
\hline $236.71(\mathrm{Al})$ & 0.000000 & 5.2363164 & $3.04 \mathrm{e}+08$ \\
\hline $256.80(\mathrm{Al})$ & 0.000000 & 4.8266319 & $7.68 \mathrm{e}+07$ \\
\hline $257.60(\mathrm{Al})$ & 0.0138938 & 4.8266319 & $2.40 \mathrm{e}+07$ \\
\hline $265.25(\mathrm{Al})$ & 0.000000 & 4.6728907 & $2.84 \mathrm{e}+07$ \\
\hline $281.62(\mathrm{Al}+)$ & 7.420704 & 11.821967 & $3.57 \mathrm{e}+08$ \\
\hline $358.66(\mathrm{Al}+)$ & 11.846618 & 15.302546 & $2.12 \mathrm{e}+09$ \\
\hline $466.30(\mathrm{Al}+)$ & 10.598336 & 13.256459 & $1.74 \mathrm{e}+08$ \\
\hline $559.33(\mathrm{Al}+)$ & 13.256459 & 15.472500 & $4.63 \mathrm{e}+08$ \\
\hline $624.34(\mathrm{Al}+)$ & 13.076728 & 15.062034 & $7.77 \mathrm{e}+08$ \\
\hline $669.87(\mathrm{Al})$ & 3.1427211 & 4.9930887 & $2.00 \mathrm{e}+06$ \\
\hline $704.21(\mathrm{Al}+)$ & 11.316595 & 13.076728 & $2.89 \mathrm{e}+08$ \\
\hline $705.66(\mathrm{Al}+)$ & 11.316595 & 13.073079 & $2.89 \mathrm{e}+08$ \\
\hline $746.83(\mathrm{~N})$ & 10.3358955 & 11.9955752 & $7.84 \mathrm{e}+07$ \\
\hline $777.20(\mathrm{O})$ & 9.1460911 & 10.7409313 & $2.58 \mathrm{e}+08$ \\
\hline $821.63(\mathrm{~N})$ & 10.3358955 & 11.8444769 & $1.36 \mathrm{e}+08$ \\
\hline $844.64(\mathrm{O})$ & 9.5213637 & 10.9888615 & $1.61 \mathrm{e}+08$ \\
\hline $868.61(\mathrm{~N})$ & 10.3259086 & 11.7528948 & $4.60 \mathrm{e}+07$ \\
\hline $877.39(\mathrm{Al})$ & 4.0216500 & 5.4343637 & $2.77 \mathrm{e}+06$ \\
\hline
\end{tabular}

component obtained (Figure 2C) mainly shows molecular bands of $\mathrm{AlO}$, as well as $\mathrm{Al}$ neutral lines to a lesser extent. Figure 2D shows the average scores compared with reference measurements. For the first component, 7 aluminum ionic lines were identified, with excitation energies between 11.82 and $15.47 \mathrm{eV}$. We observed that the temporal evolution of lines at $281.62 \mathrm{~nm}\left(E_{\mathrm{k}}=11.82 \mathrm{eV}\right)$ and $358.66 \mathrm{~nm}$ $\left(E_{\mathrm{k}}=15.30 \mathrm{eV}\right)$ was very close. We therefore chose the ionic line intensity at $281.62 \mathrm{~nm}$ as reference measurement of the decrease of ions emission. As shown on Figure 2D, the first component clearly prevails between 0 and 0.4 microsecond, and the temporal evolution of the MFICA scores is very close to that of the reference measurement. Scores decrease very quickly. This component therefore indicates the presence of $\mathrm{Al}^{+}$ions in the plasma and their rapid recombination to form neutral atoms in the first few hundreds of nanoseconds, leading to a fast decrease in their emission.

The reference measurement for the second component was performed by measuring the intensity of the neutral $\mathrm{Al}$ line at 265.25-nm over time. This component prevails between 0.4 and 1 microsecond. The evolution obtained is very similar to that of the MFICA scores, which continuously decrease from 400 nanoseconds after the laser shot because of plasma expansion and cooling.

The scores of the third component strongly decrease, reach a minimum at 0.6 microsecond, increase up to a plateau between 1 and 7 microseconds, and then decrease again with the cooling of the plasma. Although the contribution of this component is not significant before 1 microsecond, the evolution of scores might be related to the fast decrease of the continuum emission at very short delays and then to the start of the formation of $\mathrm{AlO}$ molecules. Indeed, as can be seen in 
IC1
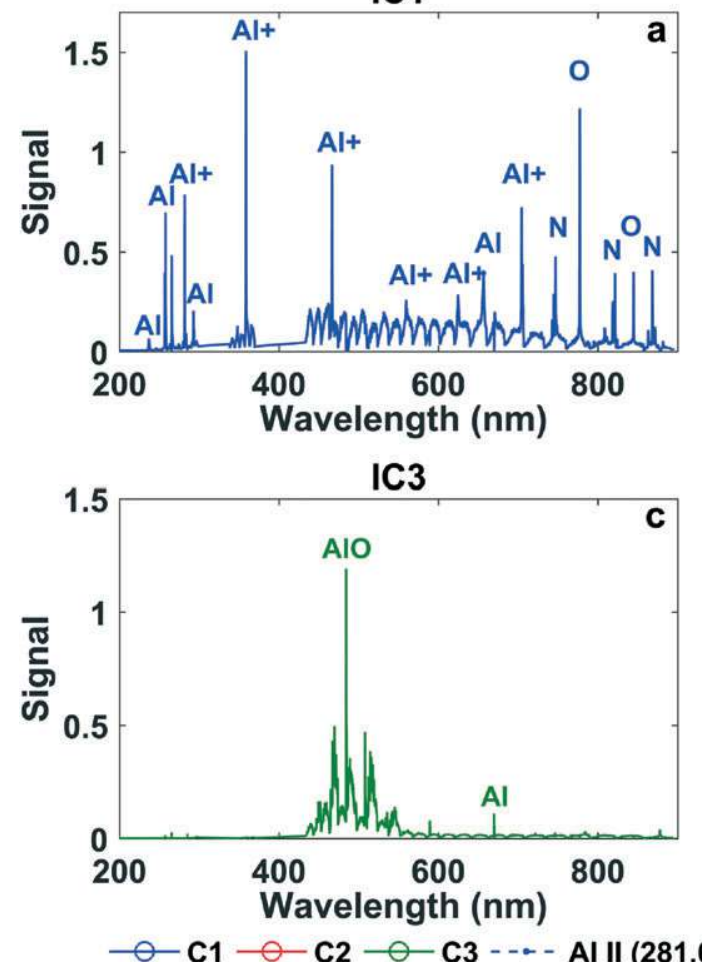
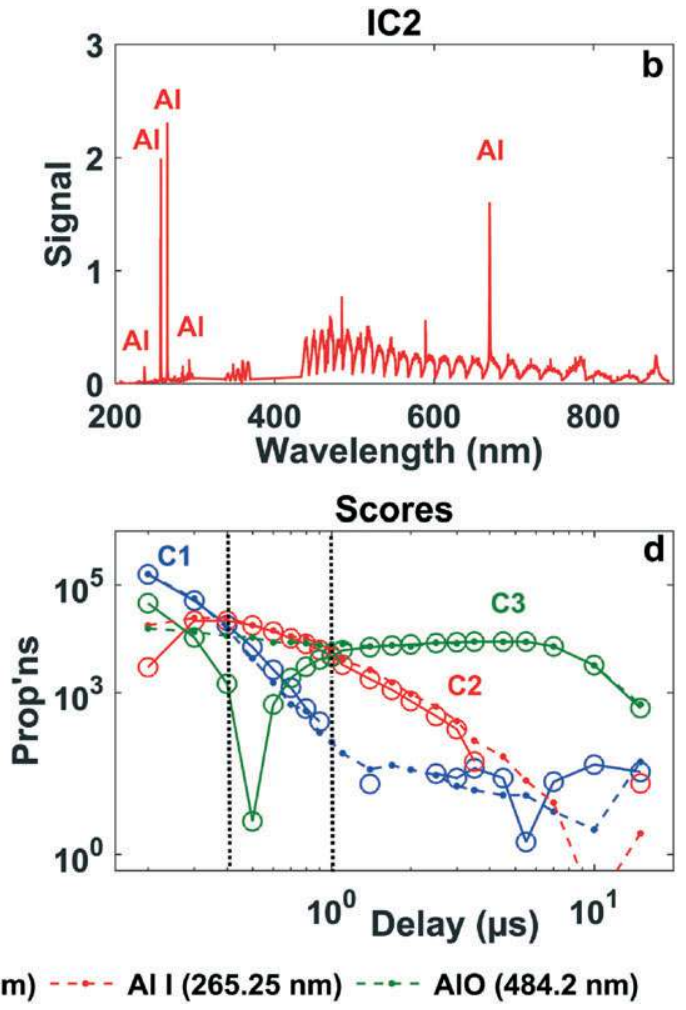

FIGURE 2 (A-C) Components obtained by mean field-independent components analysis and (D) their associated scores (open symbols) compared with reference measurements (dashed lines)

the components, the $\mathrm{AlO}$ emission spectrum has a similar shape to that of the continuum, although over a reduced spectral range, and we can assume that both contributions are simultaneously taken into account by the third component. After 1 microsecond, this component prevails. By comparing the scores to the reference method (molecular band intensity at $484.2 \mathrm{~nm}$ ), it is clear that MFICA describes well the evolution of the AlO signal in this time range. Therefore, it can be said that $\mathrm{AlO}$ molecules start to be detectable around this time. We can note that MFICA reveals information on the 2 independent phenomena occurring during the plasma lifetime, the ion recombination, and the molecular formation.

Figure 3 displays the components and scores obtained by MCR-ALS. Similarly to the MFICA results, the first component (Figure 3A) shows ionic and neutral aluminum lines with the atomic lines of oxygen and nitrogen. The second component (Figure 3B) shows the atomic lines of aluminum, oxygen, and nitrogen. In the third component (Figure 3C), only the molecular bands of $\mathrm{AlO}$ are visible. The MCR-ALS scores compared with reference measurements are represented in Figure 3D, and it is to be noted that for the 3 components the temporal evolution of the scores is very close to that of the corresponding reference measurement. We note that the scores of the third component before 1 microsecond are almost constant, which is a major difference compared to MFICA. This might be attributed to a compensation of the continuum emission decrease by the increase of the $\mathrm{AlO}$ emission. The MCR-ALS looks for pure contributions for describing the different sources of variance, so it reflects the simultaneous emission of ionic, neutral, and molecular lines. This illustrates the ion recombination because the ionic species disappear to form neutral atoms at short delays (C1), and beyond 1 microsecond the neutral atoms interact with oxygen to form emitting molecules (C3).

Overall, the ability of MFICA and MCR-ALS to describe the dataset is very similar, as shown by the good agreement between scores obtained by both methods and the univariate measurements. As mentioned previously, the main difference lies in the scores of the third component before 1 microsecond, but this occurs in a time range when the contribution of this component is negligible compared to that of the 2 others. In addition, if we consider the variance explained, we obtain an $R^{2}=.9297$ for MFICA and $R^{2}=.9274$ for MCR-ALS, confirming that both MFICA and MCR-ALS similarly fit the experimental data.

Since both methods use a non-negativity constraint for scores and components, we may conclude that using a variance criterion (MCR-ALS) or an independence criterion (MFICA) leads to an equivalent modeling of our data. Indeed, we found that the statistical independence of MCRALS and MFICA components was comparable, as illustrated by Table 3 showing the mutual information of components obtained by the 2 methods. This criterion is a measure of mutual dependence between 2 variables. It is always nonnegative, and it is zero if 2 variables are independent. ${ }^{60,61}$ The values obtained for the 2 methods are fairly similar. In addition, if we compare the results (not shown) obtained by MFICA and ICA using the JADE algorithm ${ }^{46}$ without any 

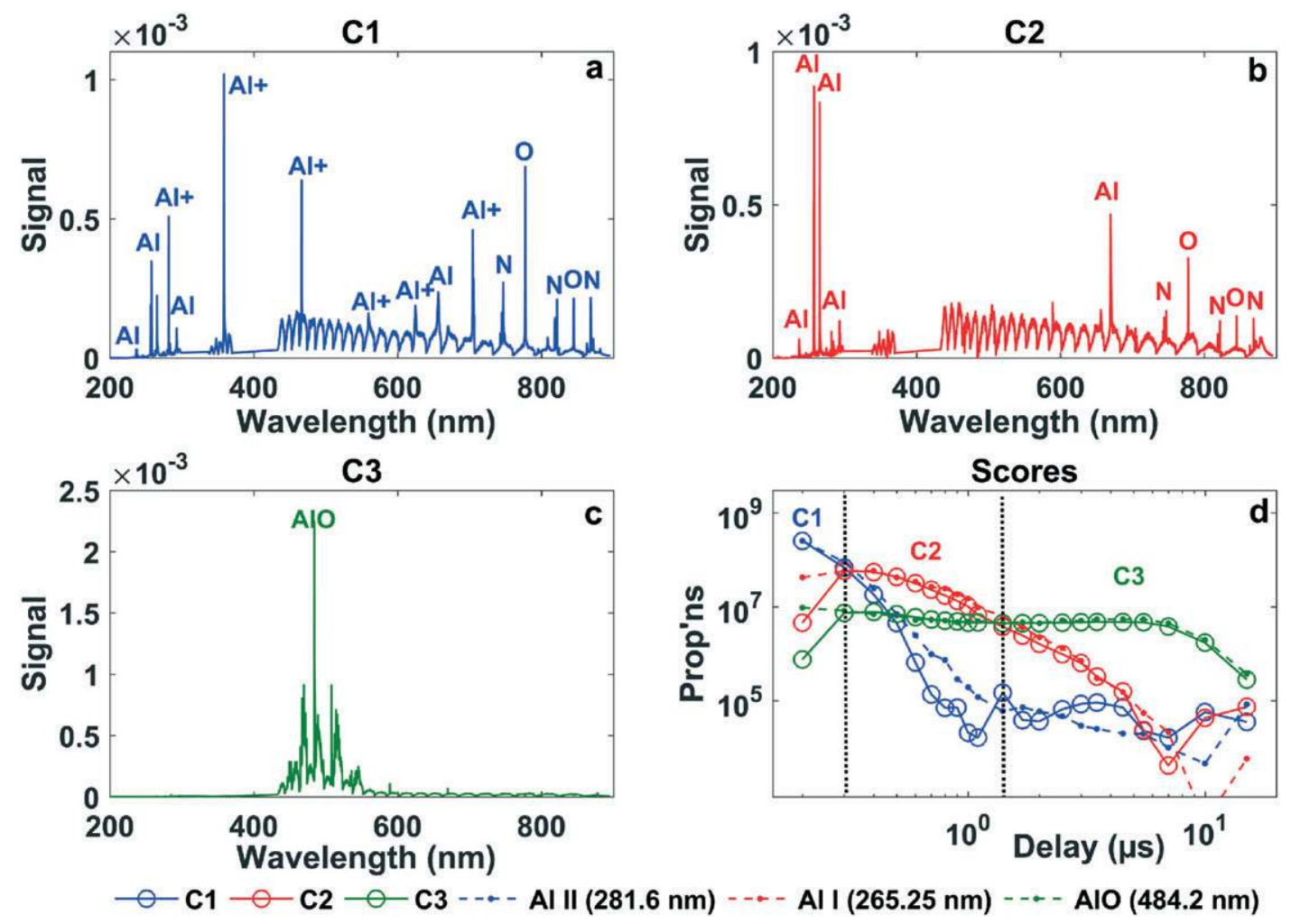

FIGURE 3 (A-C) Components obtained by multivariate curve resolution-alternating least squares and (D) their associated scores (open symbols) compared with reference measurements (dashed lines)

non-negativity constraint, we see that although MFICA better models our data, the MFICA components are less independent than those of ICA without non-negativity. This would indicate that independence of components is not a very relevant criterion to model time-resolved LIBS spectra. This might be understood if we consider that the 2 physical phenomena highlighted by the MCR-ALS and MFICA components, ion recombination and molecule formation, are not strictly speaking independent, as molecules need neutral atoms to form, which in turn need ions to form. As the number of atoms (ions and neutrals) is fixed after the end of the ablation process, population transfers occur among ions, neutrals, and molecules, and so the resulting processes are not independent.

Finally, both methods correctly model phenomena that take place between the species, and the general conclusion of this study is that in our case, MFICA solutions are equivalent to those of MCR-ALS. However, it is worth noting that introducing more constraints in the MCR-ALS model would certainly lead to a better modeling of concentration profiles

TABLE 3 Mutual information values for components obtained by MFICA and MCR-ALS

\begin{tabular}{lcc} 
& MFICA & MCR-ALS \\
\hline C1-C2 & 1.8968 & 2.0737 \\
C2-C3 & 1.2111 & 1.5448 \\
C1-C3 & 1.2889 & 1.2161 \\
\hline
\end{tabular}

Abbreviations: MCR-ALS, multivariate curve resolution-alternating least squares; MFICA,mean field-independent components analysis. and spectra. As explained previously, it was our intention to keep an unsupervised approach, as general as possible. However, MCR-ALS performances could probably be improved, for example, by using additional adequate constraints or by introducing univariate measurements as initial estimates of the scores.

\section{5 | DISCUSSION}

To the best of our knowledge, this is the first published paper describing the use of multivariate methods to treat spectrotemporal data. In the literature, the spectro-temporal LIBS data were processed and interpreted by univariate methods. Nevertheless, multivariate methods increase the accuracy, power, and efficiency of data analysis strategies compared to separate univariate methods.

Hence, Baudelet et al studied the correlation between the temporal signal of $\mathrm{C}$ and $\mathrm{CN}$ obtained on organic samples to determine whether the $\mathrm{CN}$ emission originated from native bonds or from recombination of carbon with nitrogen from the ambient air. ${ }^{62}$ However, the correlation between the $\mathrm{CN}$ and $\mathrm{N}$ signals was not studied. Similarly, Piehler et al used univariate intensity measurements of $\mathrm{AlO}$ emission under different atmospheres to be able to determine if this signal stemmed from the reaction of aluminum atoms with oxygen in the plasma or from the $\mathrm{Al}_{2} \mathrm{O}_{3}$ layer at the sample surface. ${ }^{63}$ However, the atomic oxygen signal could also have been considered to support their observation, which was not done in 
the paper. In the study of Diaz et al, ${ }^{64}$ the kinetics of successive titanium ionic species in $\mathrm{TiO}$ or $\mathrm{TiO}_{2}$ plasma were determined by time-of-flight measurements. The relatively noisy signals obtained are not straightforward to interpret, while the very complex emission spectra seem to provide a lot of information, and could have been more efficiently exploited by multivariate methods. In an aluminum plasma, Dawood et al did timeresolved measurements of the $\mathrm{Al}$ and $\mathrm{Al}^{+}$emission, of the plasma dimension, temperature, and density, for different ambient gases and pressures. ${ }^{65}$ Here again, a multivariate approach could be more suitable for such a multi-parametric study, to be able to interpret possible coupling between the different factors and responses. Finally, from an analytical point of view, several issues are essential to optimize performances, such as the choice of the emission line used for calibration, the optimization of the detection time gating, or the diagnosis of self-absorption. All those parameters are coupled, as shown by several articles, ${ }^{66-68}$ and might be efficiently studied by multivariate analyses of time-resolved spectra, using an approach similar to the one developed in this paper.

The work presented here is also related to simple spectra to be able to focus on the comparison of 2 chemometric methods, to determine their potential to describe time-resolved LIBS data, and to validate multivariate data treatment methods in relation to the usual univariate methods. The papers cited in this discussion show that these methods are indeed very promising and open the path to the interpretation of more complex spectra (multi-elemental compounds, line-rich elements, etc), and to a more precise and complete description of the plasma temporal emission, leading to a deeper understanding of the physical phenomena. Those issues will be addressed in more detail in a forthcoming article.

\section{6 | CONCLUSION}

Chemometric methods have been used for several years to analyze LIBS spectra for samples identification or for quantitative measurements. Here, MFICA and MCR-ALS are used to describe the temporal evolution of the plasma emission with an unsupervised approach. It is to be noted that the use of these methods for this purpose is new and that this approach has never been published in LIBS.

The MFICA solutions are equivalent to those of MCRALS when non-negativity constraints are applied both for scores and components. Phenomena that take place between the species and ionic recombination and formation of molecules are clearly characterized, and their temporal dynamics can be accurately determined. Differences between both methods are observed in the scores associated to the third component, but this occurs in a time range when the contribution of this component is negligible compared to that of the other 2, and overall, both MFICA and MCR-ALS describe similarly the experimental data. Admittedly, MCRALS allows to introduce more constraints in the model, which would help improving its accuracy. Yet in this work our choice was different, as we aimed at testing as general an approach as possible, hence at introducing no particular knowledge on the sample analyzed in the model calculation.

This study illustrates the strength of a multivariate approach to process time-resolved LIBS spectra. Beyond this study, we think that the interpretation of the components and scores can be used to get physical information on the plasma parameters (electron temperature and density), to optimize the choice of the analytical line, and to optimize the temporal gating used for quantitative measurements. These promising perspectives will be addressed in our future work.

\section{REFERENCES}

1. Hahn DW, Omenetto N. Laser-induced breakdown spectroscopy (LIBS), part I: review of basic diagnostics and plasma-particle interactions: still-challenging issues within the analytical plasma community. Appl. Spectrosc. 2010;64:335-366

2. Hahn DW, Omenetto N. Laser-induced breakdown spectroscopy (LIBS), part II: review of instrumental and methodological approaches to material analysis and applications to different fields. Appl. Spectrosc. 2012;66:347-419.

3. Fichet $\mathrm{P}$, Mauchien P, Moulin C. Determination of impurities in Uranium and Plutonium dioxides by laser-induced breakdown spectroscopy. Appl. Spectrosc. 1999;53:1111-1117.

4. Gallou G, Sirven JB, Dutouquet C, Bihan OL, Frejafon E. Aerosols analysis by LIBS for monitoring of air pollution by industrial sources. Aerosol Sci. Technol. 2011;45(8):918-926.

5. Syvilay D, Texier A, Arles A, et al. Trace element quantification of lead based roof sheets of historical monuments by laser induced breakdown spectroscopy. Spectrochim. Acta B At. Spectrosc. 2015;103-104:34-42.

6. Camacho JJ, Diaz L, Martinez-Ramirez S, Caceres JO. Time- and spaceresolved spectroscopic characterization of laser-induced swine muscle tissue plasma. Spectrochim. Acta B At. Spectrosc. 2015;111:92-101.

7. Lasheras R.J, Bello-Galvez C, Anzano J. Identification of polymers by LIBS using methods of correlation and normalized coordinates. Polym. Test. 2010 29(8): 1057-1064.

8. Zhang TL, Liang L, Wang K, et al. A novel approach for the quantitative analysis of multiple elements in steel based on laser-induced breakdown spectroscopy (LIBS) and random forest regression (RFR). J. Anal. At. Spectrom. 2014;29(12):2323-2329.

9. Zhang T-L, Wu S, Tang HS, Wang K, Duan YX, Li H. Progress of chemometrics in laser-induced breakdown spectroscopy analysis. Chin. J. Anal. Chem. 2015;43(6):939-948.

10. Zou XH, Guo LB, Shen M, et al. Accuracy improvement of quantitative analysis in laser-induced breakdown spectroscopy using modified wavelet transform. Opt. Express. 2014;22(9):10233-10238.

11. Yuan T, Wang Z, Li Z, Ni W, Liu J. A partial least squares and wavelet-transform hybrid model to analyze carbon content in coal using laser-induced breakdown spectroscopy. Anal. Chim. Acta. 2014;807:29-35.

12. Zhang B, Sun L, Yu H, Xin Y, Cong Z. Wavelet denoising method for laserinduced breakdown spectroscopy. J. Anal. At. Spectrom. 2013;28(12):1884

13. Schlenke J, Hildebrand L, Moros J, Laserna JJ. Adaptive approach for variable noise suppression on laser-induced breakdown spectroscopy responses using stationary wavelet transform. Anal. Chim. Acta. 2012;754:8-19.

14. Body D, Chadwick BL. Optimization of the spectral data processing in a LIBS simultaneous elemental analysis system. Spectrochim. Acta B 2001;56:725-736

15. Bohling C, Hohmann K, Scheel D, et al. Spectrochim. Acta B. 2007;62:1519-1527.

16. Sirven JB, Salle B, Mauchien P, Lacour JL, Maurice S, Manhes G.Feasibility study of rock identification at the surface of Mars by remote laser-induced 
breakdown spectroscopy and three chemometric methods. J. Anal. At Spectrom. 2007; 22:1471-1480.

17. Forni $\mathrm{O}$, Maurice $\mathrm{S}$, Gasnault $\mathrm{O}$, et al. Independent component analysis classification of laser induced breakdown spectroscopy spectra. Spectrochim. Acta B At. Spectrosc. 2013;86:31-41.

18. Gottfried JL, De Lucia FC, Munson CA Jr, Miziolek AW. Strategies for residue explosives detection using laser-induced breakdown spectroscopy. J. Anal. At. Spectrom. 2008;23:205-216.

19. Gottfried JL. Discrimination of biological and chemical threat simulants in residue mixtures on multiple substrates. Anal. Bioanal. Chem. 2011;400:3289-3301.

20. Sirven JB, Pailloux A, M'Baye Y, Coulon N, Alpettaz T, Gosse S. Towards the determination of the geographical origin of yellow cake samples by laser-induced breakdown spectroscopy and chemometrics. J. Anal. At. Spectrom. 2009;24:451-459.

21. Pease P, Tchakerian V. Source provenance of carbonate grains in the Wahiba Sand Sea, Oman, using a new LIBS method. Aeolian Res. 2014;15:203-216.

22. Moncayo S, Manzoor S, Navarro-Villoslada F, Caceres JO. Evaluation of supervised chemometric methods for sample classification by laser induced breakdown spectroscopy. Chemom. Intell. Lab. Syst. 2015;146:354-364.

23. Aquino FWB, Pereira-Filho E-R. Analysis of the polymeric fractions of scrap from mobile phones using laser-induced breakdown spectroscopy: chemometric applications for better data interpretation. Talanta. 2015;134:65-73.

24. Boucher TF, Ozanne MV, Carmosino ML, et al. A study of machine learning regression methods for major elemental analysis of rocks using laser-induced breakdown spectroscopy. Spectrochim. Acta B At. Spectrosc. 2015;107:1-10.

25. El Haddad J, Villot-Kadri M, Ismael A, et al. Artificial neural network for onsite quantitative analysis of soils using laser induced breakdown spectroscopy. Spectrochim. Acta B At. Spectrosc. 2013;79-80:51-57.

26. Li K, Guo L, Li C, et al. Analytical-performance improvement of laser-induced breakdown spectroscopy for steel using multi-spectral-line calibration with an artificial neural network. J. Anal. At. Spectrom. 2015;30(7):1623-1628.

27. Tarazona JL, Guerrero J, Cabanzo R, Mejia-Ospino E. Construction of a predictive model for concentration of nickel and vanadium in vacuum residues of crude oils using artificial neural networks and LIBS. Appl. Opt. 2012;51: B108-B114.

28. D'Andrea E, Pagnotta S, Grifoni E, et al. A hybrid calibration-free/artificial neural networks approach to the quantitative analysis of LIBS spectra. Appl. Phys. B Lasers Opt. 2015;118(3):353-360.

29. Borges FO, Cavalcanti GH, Gomes GC, Palleschi V, Mello A. A fast method for the calculation of electron number density and temperature in laserinduced breakdown spectroscopy plasmas using artificial neural networks. Appl. Phys. B Lasers Opt. 2014;117(1):437-444.

30. Bohling C, Scheel D, Hohmann K, Schade W, Reuter M, Holl G. Fiber-optic laser sensor for mine detection and verification. Appl. Opt. 2006;45:3817-3825.

31. Smolentsev GY, Soldatov AV. Analysis of time-resolved XANES spectra for determining the organometallic compound structure in solution. Journal of Surface Investigation. X-ray, Synchrotr. Neutr. Tech. 2009;3(3):398-401.

32. Zhou Y, Li B, Zhang P. Fourier transform infrared (FT-IR) imaging coupled with principal component analysis (PCA) for the study of photooxidation of polypropylene. Appl. Spectrosc. 2012;66(5):566-573.

33. Kassouf A, El Rakwe M, Chebib H, Ducruet V, Rutledge DN, Maalouly J. Independent components analysis coupled with 3D-front-face fluorescence spectroscopy to study the interaction between plastic food packaging and olive oil. Anal. Chim. Acta. 2014;839:14-25.

34. Smolders A, De Martino F, Staeren N, et al. Dissecting cognitive stages with time-resolved fMRI data: a comparison of fuzzy clustering and independent component analysis. Magn. Reson. Imaging. 2007;25(6):860-868.

35. Martin JCG, Spietz P, Orphal J, Burrows JP. Principal and independent components analysis of overlapping spectra in the context of multichannel timeresolved absorption spectroscopy. Spectrochim. Acta A. 2004;60:2673-2693.

36. Garrido M, Rius FX, Larrechi MS. Multivariate curve resolution-alternating least squares (MCR-ALS) applied to spectroscopic data from monitoring chemical reactions processes. Anal. Bioanal. Chem. 2008;390:2059-2066. doi: $10.1007 / \mathrm{s} 00216-008-1955-6$
37. Batonneau-Gener I, Moissette A, Bremard C, Buntinx G. Time resolved resonance Raman, transient diffuse reflectance and kinetic studies of species generated by UV aser photolysis of biphenyl occluded within dehydrated Yfaujasite zeolites. J. Photochem. Photobiol. A Chem. 2008;195(2-3):156-166.

38. Cassinelli WH, Martins L, Passos AR, et al. Multivariate curve resolution analysis applied to time-resolved synchrotron X-ray absorption spectroscopy monitoring of the activation of copper alumina catalyst. Catal. Today. 2014:229:114-122.

39. Debus B, Sliwa M, Miyasaka H, Abe J, Ruckebusch C. Multivariate curve resolution-alternating least squares to cope with deviations from data bilinearity in ultrafast time-resolved spectroscopy. Chemom. Intell. Lab. Syst. 2013;128:101-110.

40. Mezzetti A, Blanchet L, de Juan A, Leibl W, Ruckebusch C. Ubiquinol formation in isolated photosynthetic reaction centres monitored by time-resolved differential FTIR in combination with 2D correlation spectroscopy and multivariate curve resolution. Anal. Bioanal. Chem. 2011;399(6):1999-2014.

41. Ruckebusch C, Sliwa M, Pernot P, de Juan A, Tauler R. Comprehensive data analysis of femtosecond transient absorption spectra: a review. J Photochem Photobiol C: Photochem Rev. 2012;13(1):1-27.

42. Saad R, L'Hermite D, Bousquet B. Unexpected temporal evolution of atomic spectral lines of aluminum in a laser induced breakdown spectroscopy experiment. Spectrochim. Acta B At. Spectrosc. 2014;101:330-334.

43. Cremers DA, Yueh FY, Singh JP, Zhang H. Laser-induced breakdown spectroscopy, elemental analysis. Encycl. Analyt. Chem. 2012;1(26). doi: 10.1002/9780470027318.a0708.pub2

44. Wang G, Ding Q, Hou Z. Independent component analysis and its applications in signal processing for analytical chemistry. TrAC Trends Anal. Chem. 2008;27(4):368-376.

45. Hyva rinen A, Oja E. Independent component analysis: algorithms and applications. Neural Netw. 2000;13:411-430.

46. Rutledge DN, Jouan-Rimbaud Bouveresse D. Independent components analysis with the JADE algorithm. Trends Anal. Chem. 2013;50:22-32.

47. Rutledge DN, Jouan-Rimbaud Bouveresse D. Corrigendum to 'Independent Components Analysis with the JADE algorithm'. Trends Anal. Chem. 2015;67:220

48. Kassouf A, Maalouly J, Rutledge DN, Chebib H, Ducruet V. Rapid discrimination of plastic packaging materials using MIR spectroscopy coupled with independent components analysis (ICA). Waste Manag. 2014;34(11):2131-2138.

49. Krishnaveni V, Jayaraman S, Manoj Kumar PM, Shivakumar K, Ramadoss K. Comparison of independent component analysis algorithms for removal of ocular artifacts from electroencephalogram. Meas. Sci. Rev. 2005;5(section 2):67-78.

50. Hojen-Sorensen PADFR, Winther O, Hansen LK. Mean field approaches to independent component analysis. Neural Comput. 2002;14:889-918.

51. Lee DD, Seung HS. Learning the parts of objects by non-negative matrix factorization. Lett. Nature. 1999;401:788-791.

52. Parastar H, Jalali-Heravi M, Tauler R. Is independent component analysis appropriate for multivariate resolution in analytical chemistry? Trends Anal. Chem. 2012;31:134-143.

53. Jouan-Rimbaud Bouveresse D, Moya-Gonzalez A, Ammari F, Rutledge DN. Two novel methods for the determination of the number of components in independent components analysis models. Chemom. Intell. Lab. Syst. 2012;112:24-32.

54. Tauler R. Multivariate curve resolution applied to second order data. Chemom. Intell. Lab. Syst. 1995;30:133-146.

55. Hoecker A, Kartvelishvili V. SVD approach to data unfolding. Nucl. Instrum. Meth. A. 1996;372:469-481.

56. Maeder M, Zilian A. Evolving factor analysis, a new multivariate technique in chromatography. Chemom. Intell. Lab. Syst. 1988;3:205-213.

57. Jaumot J, De Juan A, Tauler R. Software description: MCR-ALS GUI 2.0: new features and applications. Chemom. Intell. Lab. Syst. 2015;140:1-12.

58. ICA Toolbox Homepage. http://isp.imm.dtu.dk/toolbox/ica.

59. Multivariate curve resolution homepage http://www.mcrals.info/. 
60. Bell AJ, Sejnowski TJ. An information-maximization approach to blind separation and blind deconvolution. Neural Comput. 1995;7:1129-1159.

61. Bach FR, Jordan MI. Kernel independent component analysis. J. Mach. Learn. Res. 2002;3:1-48.

62. Baudelet M, Boueri M, Yu J, et al. Timeresolved ultraviolet laser-induced breakdown spectroscopy for organic material analysis. Spectrochim. Acta B At. Spectrosc. 2007;62(12):1329-1334.

63. Piehler T, Thuvan NP, DeLucia FC Jr, et al. Temporal evolution of the laser induced breakdown spectroscopy spectrum of aluminum metal in different bath gases. Appl. Opt. 2005;44:3654-3660.

64. Diaz L, Camacho JJ, Sanz M, Hernandez M, Jandova V, Castillejo M. Temporal evolution study of the plasma induced by $\mathrm{CO} 2$ pulsed laser on targets of titanium oxides. Spectrochim. Acta B At. Spectrosc. 2013;86:88-93.

65. Dawood MS, Margot J. Effect of ambient gas pressure and nature on the temporal evolution of aluminum laser induced plasmas. AIP Adv. 2014;4: :037111. doi: 10.1063/1.4869076

66. Fisher BT, Johnsen HA, Buckley SG, Hahn DW. Temporal gating for the optimization of laser-induced breakdown spectroscopy detection and analysis of toxic metals. Appl. Spectrosc. 2001;55:1312-1319.
67. Aguilera JA, Aragon C. Characterization of laser-induced plasmas by emission spectroscopy with curve-of-growth measurements. Part I: temporal evolution of plasma parameters and self-absorption. Spectrochim. Acta B At. Spectrosc. 2008;63(7):784-792.

68. Hegazy H, Abdel-Wahab EA, Abdel-Rahim FM, Allam SH, Nossair AMA Laser-induced breakdown spectroscopy: technique, new features, and detection limits of trace elements in Al base alloy. Appl. Phys. B Lasers Opt. 2014;115:173-183. 